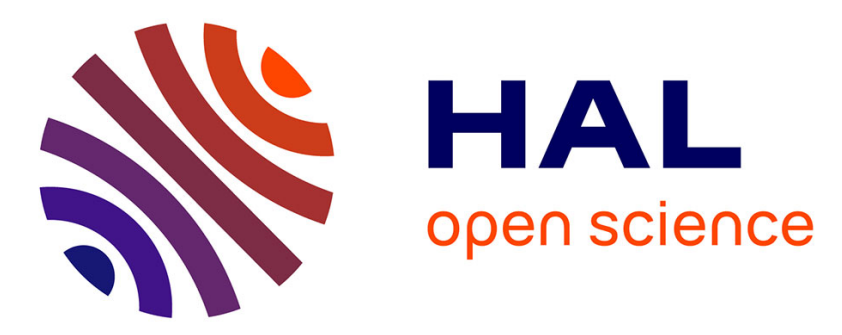

\title{
Le religieux comme objet en science politique: des recompositions de la division du travail scientifique à l'ouverture de chantiers de recherche distincts
}

Franck Frégosi, Guillaume Silhol

\section{- To cite this version:}

Franck Frégosi, Guillaume Silhol. Le religieux comme objet en science politique: des recompositions de la division du travail scientifique à l'ouverture de chantiers de recherche distincts. Mélanges de l'École française de Rome - Italie et Méditerranée, 2017. hal-01798147

\section{HAL Id: hal-01798147 \\ https://hal.science/hal-01798147}

Submitted on 23 May 2018

HAL is a multi-disciplinary open access archive for the deposit and dissemination of scientific research documents, whether they are published or not. The documents may come from teaching and research institutions in France or abroad, or from public or private research centers.
L'archive ouverte pluridisciplinaire HAL, est destinée au dépôt et à la diffusion de documents scientifiques de niveau recherche, publiés ou non, émanant des établissements d'enseignement et de recherche français ou étrangers, des laboratoires publics ou privés. 


\section{Le religieux comme objet en science politique : des recompositions de la division du travail scientifique à l'ouverture de chantiers de recherche distincts}

\section{VERSION PROVISOIRE AVANT PUBLICATION PAR LES MEFRIM}

FRANCK FRÉGOSI (professeur des universités en science politique, CHERPA, Institut d'Etudes Politiques d'Aix-en-Provence) et GUILLAUME SILHOL (doctorant en science politique, CHERPA, Institut d'Etudes Politiques d'Aix-en-Provence)

Aborder les faits religieux en science politique ${ }^{1}$, malgré la visibilité des controverses actuelles sur la compréhension de l'islam radical, n'allait pas de soi aux débuts de la discipline en France. En effet, une esquisse d'état de l'art sur la question montre plusieurs déplacements dans le temps des problématiques sur des objets «religieux», aux contours flous par excellence, et des paradoxes liés à l'histoire de la discipline et à ses relations avec des disciplines connexes, en particulier la sociologie des religions. Leur déploiement dans les sous-champs de la science politique reflète aussi les débats sur la définition de «la religion » ou du « religieux », entre approches fonctionnelles et substantives, centrées sur le croire ou sur des incidences pratiques. En conséquence, les approches des faits religieux par les politistes oscillent entre des analyses complémentaires de celles de la sociologie des religions, et d'autres proches des préoccupations généralistes de la science politique française ${ }^{2}$. Pour retracer ces déplacements, il convient de suspendre la prétention à adopter une définition de travail du « religieux », même non normative, en faveur d'un regard modérément nominaliste sur les recherches menées en la matière.

Si les débats dominants en sociologie des religions de l'après-guerre à la fin des années 1970 sur le paradigme de la sécularisation ont minoré l'étude des relations au politique ${ }^{3}$, cette tendance s'est inversée au point de favoriser la constitution dans les années 2000 d'un champ de recherches dominé par les études de l'islam politique ${ }^{4}$, et plus récemment par les 
recherches sur des phénomènes de radicalisation empruntant un référentiel islamique ${ }^{5}$. Les conflits d'analyse en la matière traduisent tant des modes différents de compréhension desdits phénomène que des rivalités liées à des positions occupées par des opérateurs du champ scientifique. En outre, à l'image des concepts de la sociologie de Pierre Bourdieu, l'usage abondant du champ lexical religieux comme analogie pour penser les rites, les clercs et les profanes du champ politique, est longtemps allé de pair avec un désintérêt pour les aspects politiques desdits faits religieux : les relations de pouvoir, la politisation d'activités cultuelles ou la gouvernementalité dans des organisations religieuses ${ }^{6}$. De même, cette valorisation paradoxale par défaut du «religieux » a conforté, plus qu'elle n'a réduit, l'isolement partiel des débats et des thématiques de la sociologie des religions, centrés sur la subjectivité au détriment des institutions, par rapport aux tendances théoriques de la sociologie généraliste ${ }^{7}$. À l'inverse, les politistes ont pu se voir reprocher, selon Danièle Hervieu-Léger, de réduire les religions à «du politique informe et de la science infirme $»^{8}$. Il convient donc de regarder avec recul les frontières disciplinaires, tant l'autonomisation des problématiques sur les faits religieux résulte de controverses publiques comme de réorganisations internes. Le présent article cible plusieurs approches développées dans la science politique française, ni exhaustives ni exclusives d'autres traditions disciplinaires, sur des faits désignés et décrits comme religieux. Les publications et les événements scientifiques en science politique offrent un « carottage » de la discipline et de ces redéploiements.

Ainsi, de premières recherches sur des faits religieux dans la décennie 1970-1980 sont conduites avec des méthodes et des problématiques propres à la science politique française, des recherches dont la genèse fait l'objet de la première partie de cet article. Toutefois, ce n'est qu'au début des années 2000, avec le redéploiement de questions généralistes de science politique sur des faits religieux, mais aussi sous la contrainte de développer une distance critique envers les controverses sur l'islam, la laïcité et les «sectes » en France, que les 
recherches de sociologie politique du religieux acquièrent une certaine autonomie vis-à-vis de la sociologie des religions. Dans une deuxième partie, nous verrons leur déploiement dans trois directions : la sociologie politique de la laïcité, la sociologie des mobilisations à référents religieux, et la sociologie du religieux dans l'action publique. Ces développements permettront d'esquisser une conclusion sur leurs acquis et leurs limites, qui conservent des angles morts dans les problématiques et les objets.

Les recherches sur les faits religieux en science politique française, de l'analogie à des objets initialement périphériques

La construction d'objets de recherche sur des faits religieux en science politique française, comme dans d'autres traditions disciplinaires jusqu'à la fin du XXe siècle ${ }^{9}$, se produit au gré des recompositions des sous-branches de la discipline, la sociologie politique, les politiques publiques, les relations internationales et l'histoire des idées politiques. Avant les années 1970, à rebours des lectures et des usages de Max Weber, Emile Durkheim ou d'autres «pères fondateurs » de la sociologie, l'intérêt pour le religieux en science politique reste cantonné aux travaux d'histoire des idées, de théorie politique voire de théologie politique sous influence anglo-saxonne. Dans le contexte des débats sur le paradigme classique de la sécularisation en sociologie des religions, le religieux n'est alors pas un «sujet d'avenir» mais un fonds conceptuel pour penser le politique. Ces travaux, issus de réflexions de la première moitié du XXe siècle et inspirés par des auteurs aussi opposés qu'Ernst Kantorowicz $^{10}$, Carl Schmitt ${ }^{11}$, Eric Voegelin ${ }^{12}$ ou Robert Bellah ${ }^{13}$, reposent sur l'idée d'une analogie entre une structure théologique, de matrice judéo-chrétienne, et une structure politique formellement indépendante. L'analogie est mobilisée pour penser les « religions séculières » ou «religions politiques » comme le communisme, non sans critiques sur 
l'assimilation de l'idéologie à la religion, par exemple dans les réponses d'Hannah Arendt au sociologue Jules Monnerot, auteur en 1949 d'un essai de Sociologie du communisme. Echec d'une tentative religieuse ${ }^{14}$. Une partie des travaux de Raymond Aron sur les idéologies, avant l'autonomisation de la science politique en discipline universitaire, reflète aussi ces débats ${ }^{15}$.

Après l'étape fondatrice du divorce avec le droit public et de l'instauration en 1973 d'une agrégation en science politique ${ }^{16}$, les faits religieux abordés sont principalement construits comme des facteurs électoraux dans la sociologie des comportements de vote, ou des cultures politiques dites traditionnelles. La sociologie politique française est alors influencée par la politologie et la sociologie américaines dominées par Paul Lazarsfeld, tandis que l'analyse des politiques publiques assimile les travaux anglo-saxons et la sociologie des systèmes d'action concrets de Michel Crozier. Cette orientation rationaliste conduit à marginaliser les réflexions de philosophie politique, par rapport aux sciences administratives et à la sociologie de 1'Etat. Ainsi, l'historien du droit Pierre Legendre fustige en 1976 un impensé tant du religieux que du symbolique, dans une science politique française « radicalement théocratique, respectueuse de l'idéalisation centraliste ${ }^{17}$. » Toutefois, cette critique ignore les balbutiements de l'intérêt pour le religieux comme une variable dans d'autres recherches, qui commencent à étudier les croyances et les appartenances religieuses en sociologie des comportements électoraux. Les travaux pionniers de Guy Michelat et Michel Simon sur les rapports entre religion, classe et vote, démontrent ainsi les relations fortes entre socialisation religieuse et vote conservateur. S'appuyant sur une méthodologie mixte d'entretiens non-directifs et d'enquête par questionnaire, ils notent un rapport de proximité entre une intégration forte à la vie religieuse catholique (observance religieuse régulière, participation aux activités paroissiales...) et la probabilité d'expression d'un vote pour des partis de droite, là où l'indifférence religieuse semble plus favorable aux partis de gauche ${ }^{18}$. Ces tendances lourdes contribuent à pérenniser 
les enquêtes qui construisent la religion, sous forme d'appartenance, de croyances ou de pratiques catégorisées et déclarées, comme une variable indépendante en sociologie politique des élections. Elles sont cependant nuancées par l'infirmation des hypothèses d'un vote confessionnel homogène, dans des études attentives à d'autres facteurs dans l'approche écologique du vote, puis par d'autres problématiques. Dans cette lignée, les travaux ultérieurs de Claude Dargent posent la question des variables complexes dans les comportements d'un « segment électoral musulman », dans des zones urbaines déclassées et votant à gauche ${ }^{19}$.

\section{Le religieux abordé par les marges de la sociologie politique}

Pour leur part, les recherches qualitatives sur la politisation des expressions religieuses et de l'imaginaire religieux ne se trouvent alors qu'aux marges de la sociologie des religions en France, comme la thèse de doctorat de Danièle Hervieu-Léger sur le militantisme des étudiants catholiques ${ }^{20}$. En ce sens, l'intérêt pour des recherches en science politique française à l'écart de l'explication d'un corpus théologique, et distinctes du noyau dur des sciences sociales des religions, n'apparaît qu'à la fin des années 1970. Il résulte des tentatives d'interpréter des phénomènes divers, comme la révolution iranienne ou le mouvement Solidarność, qui contredisent le paradigme classique de la sécularisation comme processus d'affaiblissement généralisé du religieux. La désinstitutionnalisation du religieux traditionnel et l'émergence de nouvelles mobilisations, en Europe comme ailleurs, rendent nécessaire l'analyse de leurs incidences politiques et de mouvements arborant des référents religieux explicites, sans revenir à d'anciennes définitions ${ }^{21}$. Ces phénomènes ne sont pas non plus explicables par les cultures politiques et les clivages traditionnels, entre cléricaux, socialistes et libéraux, mis en exergue par la sociologie des partis ${ }^{22}$. Or, leur apparition dans l'espace public et la circulation de débats coïncide avec les recompositions de la science politique 
française. Sous l'influence de la sociologie critique et d'un recours systématisé à l'enquête empirique au détriment de la théorie politique, ces changements confèrent davantage de légitimité scientifique aux regards des politistes sur les faits religieux, au-delà d'un registre d'analogies. Le «tournant historique » entrepris au cours des années 1980 dans les sciences sociales françaises ${ }^{23}$, réverbéré dans la science politique en voie de «sociologisation », favorise les efforts interdisciplinaires entre des politistes tels Alain Garrigou ou Michel Offerlé et des historiens comme Gérard Noiriel, avec la revue Genèses. Il contribue en parallèle à la diffusion des thèses de Marcel Gauchet dans Le désenchantement du monde, sur le religieux et le politique comme domaines en constante interaction ${ }^{24}$. Ainsi, bien qu'aucun chapitre ne porte sur le «religieux » dans le Traité de science politique dirigé par Madeleine Grawitz et Jean Leca en 1985, les thématiques religieuses sont récurrentes dans les contributions de Jacques Lagroye sur la légitimation, de Raphaël Draï sur la psychanalyse ou de Pierre Birnbaum sur l'Etat ${ }^{25}$.

En ce sens, la formation de mouvements associés à l'islam politique, comme les mobilisations de protestants évangéliques aux Etats-Unis, offrent l'opportunité de problématiser différemment le religieux en recomposition à la fin de la Guerre froide. La première génération de recherches en sociologie politique du religieux, menée à partir de problématiques issues de la sociologie politique, apparaît alors autour de deux chantiers, la sociologie des mobilisations et la sociohistoire de la citoyenneté. Les mouvements politiques à référents religieux commencent à être étudiés en-dehors de la sociologie des clivages, chez Bruno Etienne et Rémy Leveau, agrégés de science politique, François Burgat ${ }^{26}$, Gilles Kepel et Olivier Roy sur les mouvements islamistes dans le monde arabe ${ }^{27}$. Après des premiers travaux sur le socialisme en Algérie, les recherches de Bruno Etienne contribuent à construire l'objet « islam politique » dans le monde arabe comme un objet légitime des études en science politique, et à faire de l'objet « religion » un objet à part entière et non totalement à part ${ }^{28}$. Le 
terme « islamisme » apparait ainsi dans son acception contemporaine dans le cadre de ses enquêtes au Maghreb, désignant des mouvements qui procèdent à une lecture politique de l'islam et de ses sources scripturaires ${ }^{29}$. Un phénomène homogène en apparence comme «l'islamisme », étudié dans divers contextes géographiques et sociaux, se révèle hétérogène a posteriori. Ainsi, les courants dits salafistes, malgré une apparente standardisation de la pratique et une idéalisation utopique de l'islam des origines, peuvent déboucher sur des expressions très variables, les unes religio-centrées, volontiers sectaires et politiquement abstinentes, là où d'autres courants passent au politique sur un mode partisan ou autour d'enjeux locaux, outre des formes plus radicales et violentes désignées comme « djihadistes ». La sociologie politique des mouvements islamistes, menée par exemple par Gilles Kepel sous la forme d'une généalogie et d'une analyse de corpus doctrinaux sur les Frères musulmans en Egypte $^{30}$, mais également les premiers travaux sur l'islam des populations immigrées en Europe $^{31}$, développent ainsi un filon de recherches spécifiques de science politique. Comme le remarque Vincent Geisser, qui prend part à ces premières recherches, l'investissement des politistes sur l'islam supplée à un désintérêt notoire des sociologues des religions, et aux travaux antérieurs d'islamologues arabisants comme Maxime Rodinson et Jacques Berque ${ }^{32}$. De même, les travaux de Patrick Michel sur le catholicisme polonais, les répressions et les mobilisations du religieux dans le régime communiste, contribuent à dé-singulariser l'objet « religion » dans la discipline. S'appuyant sur l'anthropologie du croire de Michel de Certeau, il montre que le religieux opère comme un répertoire symbolique parmi d'autres dans un espace de luttes, en contexte de délégitimation des institutions politiques ${ }^{33}$. À ce titre, les activités entre 1992 et 1998 du Groupe Religion, démocratie, démocratisation de l'Association Française de Science Politique (AFSP), autour de Patrick Michel et de sociologues travaillant sur l'islam iranien comme Farhad Khosrokhavar ou sur la théologie de la libération au Brésil comme Michaël Löwy, contribuent à pérenniser ces questionnements. 
Ces travaux n'amorcent pas encore une coupure vis-à-vis des débats de la sociologie des religions en France, qui connaît des recompositions liées à des désaccords sur la place de l'interdisciplinarité ou des objets de spécialisation ${ }^{34}$. En effet, à Paris, le GSRL (Groupe de Sociologie des Religions et de la Laïcité, GSRL) à l'EPHE connaît une scission au début des années 1990 et le départ de sociologues dont Danièle Hervieu-Léger vers le CEIFR (Centre d'Etudes Interdisciplinaires des Faits Religieux) à l'EHESS ${ }^{35}$, tandis que d'autres recherches sont menées par des sociologues et des politistes basés à Lyon, à Strasbourg ou encore à Aixen-Provence. En outre, ces travaux sur des mouvements contemporains poursuivent une logique de spécialisation professionnelle sur un objet religieux, une «tradition » ou une aire culturelle, à l'exception de politistes comme Jacques Palard ${ }^{36}$, Françoise Lorcerie $^{37}$ ou JeanMarie Donegani qui continuent à travailler sur d'autres thèmes comme le développement territorial, les politiques scolaires et la démocratie.

\section{Le religieux et la citoyenneté en sociohistoire du politique}

Un deuxième type de recherches touchant au religieux se déploie dans la sociohistoire de la citoyenneté, autour de Pierre Birnbaum et d'Yves Déloye. Les recherches de Pierre Birnbaum en sociologie historique du judaïsme, de l'antisémitisme et de la construction de la citoyenneté dans la République française, s'inscrivent à la fois dans la continuité de sa sociologie de l'Etat, attentive au code culturel et aux élites, et dans une rupture méthodologique par le recours privilégié à l'enquête sur $\operatorname{archives}^{38}$. Les recherches d'Yves Déloye poursuivent l'étude de la construction conflictuelle de la citoyenneté, à travers le militantisme des milieux catholiques français durant la IIIe République, dans le cléricalisme électoral et dans les «guerres scolaires ${ }^{39}$. Dans une approche différente en sociologie de la citoyenneté, les recompositions politiques des milieux catholiques français en contexte de 
sécularisation avancée, font l'objet des travaux de Jean-Marie Donegani. Dans La liberté de choisir parue en 1993, il analyse au travers d'enquêtes qualitatives les redéfinitions et les appropriations d'un pluralisme politique par des catholiques français. À partir d'une typologie des modes d'identification au catholicisme, il conteste l'idée d'une permanence de l'intransigeantisme catholique comme facteur déterminant d'orientation politique, pour souligner les effets de «la transformation interne du catholicisme et [d'une] évolution conjointe des mentalités qui ont permis que le religieux se privatise et que nombre d'attitudes touchant à la morale personnelle et au choix politique échappent à la dictée magistérielle ${ }^{40}$. » Ainsi, la première génération de travaux de politistes français sur les faits religieux, initiée sur des objets périphériques, se rapproche progressivement des questionnements centraux de la discipline, des élections aux relations de pouvoir. Ces différents types de recherches, qui continuent à nourrir des problématiques complémentaires de celles de la sociologie et de l'histoire des religions, subissent toutefois la mise en visibilité accrue du religieux dès les années 1990. Les thèmes du «religieux public», en écho aux travaux de sociologues américains comme José Casanova, font ainsi l'objet d'un colloque commun de l'Association française de sociologie des religions (AFSR) et de l'AFSP en 1999. Des politistes comme Pierre Birnbaum et Jean-Marie Donegani, mais également Christine Pina, auteure d'une thèse sous la direction de Pierre Bréchon sur la politisation des catholiques charismatiques, y interviennent sur le judaïsme et le catholicisme, tandis que les interventions sur les «autres » minorités religieuses relèvent de sociologues des religions ${ }^{41}$.

\section{Les trois chantiers spécifiques de la sociologie politique du religieux}

À la fin des années 1990, les controverses sur la laïcité, les « sectes » et l'islam favorisent un redéploiement des recherches sous contrainte. En particulier, les termes des débats et les 
commandes institutionnelles, qui imposent des problématiques sécuritaires dans le cas de l'islam et pathologiques dans le cas des $«$ sectes $»^{42}$, s'accompagnent d'un volume accru de publications d'acteurs experts, d'ONG et de fondations privées en concurrence avec les chercheurs universitaires $^{43}$. Le renouvellement de la sociologie politique du religieux s'effectue donc, notamment dans l'après-11 septembre 2001, à la fois avec et contre ce regain

d'intérêt institutionnel pour les faits religieux. À rebours de la philosophie politique anglosaxonne (Jürgen Habermas, Charles Taylor...), la science politique française investit comparativement peu les thèmes de la spiritualité et du «post-séculier ${ }^{44}$. Le renouvellement des recherches sur les faits religieux dans la science politique française est plutôt favorisé par l'approfondissement d'une sociologie politique attentive aux institutions ${ }^{45}$. Paradoxalement, si l'institutionnalisation de la discipline et des problématiques généralistes sur les partis, les mobilisations ou la citoyenneté s'était effectuée dans les années 1970 dans un sens rationaliste contre la philosophie politique, la sociologie politique contribue désormais à réinterroger les faits religieux. Ces travaux peuvent être présentés selon leur déploiement en sociologie politique de la laïcité, en sociologie des mobilisations, et en sociologie de l'action publique.

\section{Le religieux coextensif à l'Etat, une sociologie politique de la laïcité}

En prolongement des débats internes à la sociohistoire de l'Etat, l'entrée des politistes sur le terrain de l'analyse des relations Eglises-Etats, plus familier aux historiens et aux juristes, commence en complément des réflexions de quelques sociologues des religions comme Emile Poulat, Jean Baubérot et Jean-Paul Willaime ${ }^{46}$. L'enjeu réside dans le développement d'un discours sociologique sur la laïcité dans le gouvernement du religieux, autre que celui des acteurs et des institutions ${ }^{47}$. Au croisement de la théorie politique et d'une sociologie historique du religieux, les recherches de Philippe Portier problématisent le politique et le 
religieux à la fois comme des espaces sociaux et des domaines de l'imaginaire en interaction. D'une part, cette approche éclaire la question des rapports contradictoires de l'Eglise catholique à la modernité politique par l'analyse des discours de Jean-Paul II et de la formation d'une pensée politique pontificale davantage en continuité qu'en rupture avec l'intransigeantisme de la fin du XIXe siècle ${ }^{48}$. D'autre part, les pratiques de séparation et les dispositifs de reconnaissance des cultes sont étudiés par Philippe Portier comme des instruments de gouvernement, qui se recomposent et qui circulent entre les pays européens, contribuant à dé-singulariser la laïcité comme objet d'analyse en-dehors de perspectives téléologiques ${ }^{49}$. De même, l'approche du politiste François Foret contribue à la sociologie politique du religieux dans les institutions de l'Union Européenne, par l'enquête sur les manières dont «une démocratie très réflexive mettant constamment en doute ses propres fondements intègre dans son fonctionnement un discours religieux qui s'articule autour d'un sacré irréfragable ${ }^{50}$.». À partir d'une sociologie de la légitimation des institutions européennes, ses travaux interrogent les redéfinitions et l'enrôlement du religieux dans les pratiques politiques, des controverses sur les « racines chrétiennes de l'Europe » à d'autres enjeux inscrits à l'agenda politique ${ }^{51}$. Ces recherches donnent notamment lieu à un atelier du Congrès de l'AFSP de 2007 supervisé avec Xabier Itçaina et un dossier de la revue Politique européenne, dont les contributions interrogent les jeux d'acteurs informels, les discours et les négociations qui mobilisent le religieux comme ressource, sans aboutir nécessairement à des formes de convergence dans l'action publique entre les pays européens ${ }^{52}$.

\section{Le religieux mobilisé et administré, une sociologie de l'institution}

Pour leur part, les recherches centrées sur les faits religieux dans les mobilisations connaissent au cours des années 2000 un renouvellement principalement à rebours des commandes 
institutionnelles et de certaines approches antérieures. L’isolement initial des débats entre des politistes spécialistes sur l'islamisme, le post-islamisme (Olivier Roy) ou les recompositions complexes de ces mouvements (François Burgat), vis-à-vis d'autres recherches généralistes, n'empêche pas un essor et une internationalisation des travaux scientifiques sur des faits religieux dans les publications et les événements de la science politique française dans les années 2000. Valérie Amiraux évoque en ce sens un «tournant juridique » dans la sociologie politique de l'islam, en syntonie avec la reconsidération des institutions, de la gouvernance et des usages du droit en sociologie politique française ${ }^{53}$. À titre d'exemple, lors du Congrès de l'AFSP de 2007, outre l'atelier «Dieu loin de Bruxelles », trois autres ateliers abordent directement des questions religieuses. Le premier, supervisé par Camille Froidevaux-Metterie et Ariane Zambiras, porte sur «Religion et politique en démocratie : comment la laïcité résiste à l'esprit de religion », et deux autres ateliers, respectivement supervisés par Solenne Jouanneau et Julie Pagis, et par Magali Della Sudda et Yann Raison du Cleuziou, abordent les logiques de 1 'engagement et les carrières militantes religieuses ${ }^{54}$. Ces nouvelles perspectives sont liées à la diffusion des outils des études de genre et aux réflexions en sociologie des institutions autour de Jacques Lagroye.

Dans ses derniers travaux, Jacques Lagroye propose ainsi d'aborder l'Eglise catholique en France et les dispositifs paroissiaux, diocésains, associatifs, etc., qui la composent, comme une institution au sens sociologique, soit un agencement collectif perdurant dans le temps, faisant l'objet de légitimations, de critiques, d'investissements de la part d'acteurs sociaux et contribuant en retour à façonner leurs manières d'agir socialement. Cette recherche conduit d'abord à objectiver les manières dont la « crise » des institutions catholiques est socialement construite après le Concile Vatican II à partir des luttes entre deux régimes de vérité, le régime des certitudes et le régime des témoignages ${ }^{55}$. Ensuite, la sociologie du catholicisme, qui pointe des similitudes avec la sociologie des administrations ou des partis, conduit à 
prendre au sérieux les trajectoires multiples des acteurs qui habitent l'institution, leurs logiques religieuses et non-religieuses ${ }^{56}$. Sur des bases très différentes de celles de Philippe Portier, l'approche de Jacques Lagroye conduit pourtant à reconsidérer également les discours et les textes de doctrine en tant que matériaux empiriques, qui, bien que distincts des dispositifs de culte et des pratiques des acteurs, n'informent pas moins sur les relations de pouvoir et sur le politique.

Au croisement de cette sociologie de l'institution, de la sociohistoire de la citoyenneté et de la sociologie politique du genre, les travaux de Magali Della Sudda posent la question des formes de politisation et d'accès à la citoyenneté informelles, notamment dans le cas du militantisme de femmes catholiques françaises et italiennes au début du XXe siècle. La requalification d'activités familiales et associées à la «reconquête religieuse » par des femmes catholiques contribue à subvertir les rapports sociaux de sexe à l'intérieur des institutions catholiques, et leur analyse montre la complexité des pratiques d'accès à la politique des femmes, entre rigidification et transgression des normes ${ }^{57}$. Ces réflexions sont prolongées notamment dans un numéro de Genèses codirigé par Yann Raison du Cleuziou et Solenne Jouanneau en 2012, qui propose d'étudier les formes d'autorités religieuses en train de se faire ${ }^{58}$. Dans le même ordre de questionnements, l'on peut citer les travaux de Yann Raison du Cleuziou sur les formes paradoxales de politisation des dominicains français dans les années 1960, ou encore les recherches sociohistoriques de Sara Teinturier sur l'enseignement catholique ${ }^{59}$. Dans ce champ de recherches entre également l'enquête ethnographique d'Ariane Zambiras sur la politisation des communautés chrétiennes contemporaines en Californie, qui montre l'importance des imaginaires religieux dans les motifs et les pratiques politiques ordinaires des individus ${ }^{60}$. 


\section{Le religieux gouvernable, une sociologie du religieux dans l'action publique}

Enfin, le redéploiement des recherches en science politique sur des faits religieux sous l'influence de controverses contribue à banaliser l'idée de la construction de faits religieux en problèmes sociaux et en objets de politiques publiques. Ainsi, Danièle Hervieu-Léger exprime dès 2000 les enjeux de l'ouverture d'un chantier de recherche spécifique, distinct de ceux de la sociologie des religions sur les croyances et pratiques, sur les conséquences des «phénomènes de déstabilisation des dispositifs juridico-politiques de gestion de la religion dans l'espace public ${ }^{61}$. » L'utilisation de méthodes de l'analyse des politiques publiques sur des objets habituellement «étrangers », comme des pratiques cultuelles ou des normes alimentaires dans des institutions publiques, ne va initialement pas sans incompréhensions ni critiques d'un oubli du temps long de $1^{\prime} E t_{a t}{ }^{62}$. À la suite de travaux sur les «sectes » et le multiculturalisme à la fin des années 1990 au Canada ${ }^{63}$, ces recherches en science politique française portent d'abord sur des terrains locaux : le religieux dans la commune, avec un volume dirigé par Jean-Paul Willaime et Franck Frégosi ${ }^{64}$, et les travaux de Claire de Galembert sur la cathédrale d'Evry et sur l'institutionnalisation de l'islam ${ }^{65}$. Un colloque sur les politiques publiques du religieux en Europe et dans le bassin méditerranéen est ainsi organisé à l'IEP d'Aix-en-Provence en 2014, autour de recherches sur la bureaucratisation du croire $^{66}$ et sur le gouvernement de la religion dans les prisons ${ }^{67}$.

Ces questionnements sont également investis dans un sens nominaliste par des travaux de sociohistoire du religieux comme catégorie d'action publique ${ }^{68}$, notamment dans les thèses d'Etienne Ollion sur la requalification déviante de la catégorie « secte ${ }^{69}$, et de Solenne Jouanneau sur les imams en France. Cette dernière recherche, qui associe une enquête sur des fonds d'archives du Ministère de l'Intérieur à une ethnographie de l'autorité d'imam dans les mosquées, contribue à étudier la laïcité et le gouvernement du religieux dans des pratiques 
informelles notamment de politique migratoire. Elle montre comment la fonction d'imam, considérée influente par les administrations de l'Etat, est investie par des intérêts divers et comment des mesures éparses de contrôle constituent des « faisceaux d'action publique ${ }^{70}$. À cet égard, le religieux dans l'action publique fait l'objet de deux sections thématiques au Congrès de 1'AFSP de 2013, l'une sur La laïcité comme répertoire d'action administrative supervisée par Solenne Jouanneau et Yann Raison du Cleuziou, et l'autre sur La participation politique des musulmans dans les sociétés occidentales sous la direction de Julien O'Miel et Julien Talpin ${ }^{71}$. Cette dernière reprend les réflexions issues d'une enquête sur la mise en politique du projet de construction de la Grande Mosquée de Florence au début des années 2010 dans un dispositif participatif, entre des tentatives de domestication du dossier par l'expertise et une circulation de controverses au-delà de la Toscane ${ }^{72}$. Enfin, un numéro de la Revue française de science politique consacré à la judiciarisation du religieux, des actions collectives aux controverses dans les tribunaux, paraît à l'automne 2014 ${ }^{73}$. En ce sens, l'intégration d'objets dits religieux dans la sociologie de l'action publique constitue aussi une contribution notable aux problématiques générales de la discipline.

\section{Acquis et limites des regards des politistes français sur les faits religieux}

Sur quarante ans de recherches sur des faits religieux en science politique française, plusieurs acquis et quelques limites peuvent être résumés. En premier lieu, le religieux est passé d'un registre d'analogies à un objet pris au sérieux en-dehors de l'histoire et de la sociologie des religions, étudié par des méthodes généralistes aussi bien en sociologie des partis, qu'en sociologie du vote ou en politiques publiques. Ensuite, ces problématisations ont permis un essor de la sociologie politique du religieux, au détriment des relations internationales ou de la philosophie politique comme dans d'autres pays. En ce sens, l'autonomisation partielle des 
problématiques de ces recherches vis-à-vis de celles de la sociologie des religions traduit aussi une méconnaissance mutuelle avec la sociologie des religions, qui a privilégié récemment l'étude du religieux informel et de la spiritualité ${ }^{74}$. À l'inverse, la focalisation d'une partie des recherches des politistes, notamment dans les années 1990 et 2000, sur un religieux «problématique », en particulier sur les controverses sur l'islam et sur la laïcité, a posé de nouvelles contraintes et imposé des objets. La quasi-absence de travaux de politistes sur des faits religieux autres que les «trois monothéismes », judaïsme, christianisme et islam, est symptomatique de limites, tant dans la légitimité d'objets minoritaires ou moins problématiques que dans l'autonomisation précaire vis-à-vis des commandes institutionnelles. La prédominance d'études centrées sur les niveaux locaux et nationaux, au détriment des questions de la circulation des politiques publiques et de la transnationalisation des réseaux d'acteurs $^{75}$, laisse des impensés dans le champ de recherches, telles des controverses sur la laïcité qui se construisent différemment ou circulent dans nombre d'autres pays européens ${ }^{76}$. Néanmoins, cette focalisation de la sociologie politique du religieux n'est pas uniquement française : une étude quantitative menée en 2008 sur les publications ayant pour objet principal «la religion» dans des revues de science politique anglo-saxonnes depuis 2000 indiquait $21,7 \%$ d'articles sur la violence et près d'un autre cinquième sur l'islam ${ }^{77}$.

En ce sens, la réflexion théorique et empirique sur les conséquences des commandes institutionnelles, de la demande et de la production de savoirs sur des faits religieux, reste sectorielle $^{78}$. Les débats actuels sur les manières d'étudier la radicalisation ne sont qu'un exemple de cette reconfiguration. Toutefois, les travaux sur le «religieux» en science politique française montrent un certain nombre d'acquis dans les enquêtes, qui permettent de dénaturaliser et dé-spécifier le religieux problématique et les controverses publiques, tout comme les mobilisations à référents religieux inscrites dans la modernité politique. Ils constituent une réponse résolument empirique, généraliste et attentive aux configurations 
d'acteurs, aux critiques de la réification des relations entre religion et politique formulées par les approches discursives comme par certains travaux de sociologie des religions. Enfin, ils participent d'une compréhension du croire et du religieux comme des faits sociaux, des relations, des ressources et des pratiques inscrits dans un contexte historique et faisant l'objet d'investissements divers de la part d'acteurs, et de toute institution, religieuse ou non, qui « se porte garante du croire et $[\ldots]$ l'utilise à des fins sociales ${ }^{79}$. » 


\section{Bibliographie}

AFSP 2007 = AFSP, IXe Congrès de l'Association Française de Science Politique, Toulouse, 2007, documents accessibles en ligne le 07/01/2017 sur http://www.afsp.mshparis.fr/congres2007/index.html.

AFSP 2013 = AFSP, XIIe Congrès de l'Association Française de Science Politique, Paris, 2013, documents accessibles en ligne le 07/01/2017 sur http://www.afsp.info/congres2013/index.html.

Altglas $2010=$ V. Altglas, Laïcité Is What Laïcité Does. Rethinking the French Cult Controversy, dans Current Sociology, Vol. 58, N³, 2010, p. 489-510.

Altglas $2012=\mathrm{V}$. Altglas, La religion comme symptôme, dans P. Gonzalez et C. Monnot (dir.), Le religieux entre science et cité. Penser avec Pierre Gisel, Genève, 2012, p. 33-48.

Amiraux 2012a $=$ V. Amiraux, Religion and Political Sociology, dans S. Scott, E. Amenta et K. Nash (dir.), The Wiley-Blackwell Companion of Political Sociology, Malden, 2012, p. 336346.

Amiraux 2012b = V. Amiraux, État de la littérature. L'islam et les musulmans en Europe : un objet périphérique converti en incontournable des sciences sociales, dans Critique internationale, Vol. 3, N56, 2012, p. 141-157.

Aron $1965=$ R. Aron, Démocratie et totalitarisme, Paris, 1965.

Balas 2013 = M. Balas, Le pluriel «civilisé » : Sant'Egidio, une grammaire pluraliste ?, dans A.-S. Lamine (dir.), Quand le religieux fait conflit. Désaccords, négociations ou arrangements, Rennes, 2013, p. 201-211.

Baubérot - Milot 2011 = J. Baubérot et M. Milot, Laïcités sans frontières, Paris, 2011.

Baubérot 2014 = J. Baubérot, Une si vive révolte, Paris, 2014. 
Beaugé - Hajjat 2014 = J. Beaugé et A. Hajjat, Elites françaises et construction du «problème musulman». Le cas du Haut Conseil à l'Intégration (1989-2012), dans Sociologie, 2014, $\mathrm{N}^{\circ} 1$, Vol. 5, p. 31-59.

Bellah 1973 = R.N. Bellah, La religion civile en Amérique, dans Archives de sciences sociales des religions, Vol. 18, N³5, 1973, p. 7-22.

Béraud - De Galembert - Rostaing 2016 = C. Béraud, C. De Galembert et C. Rostaing, De la religion en prison, Rennes, 2016.

Birnbaum 1985 = P. Birnbaum, L'action de l'Etat. Différenciation et dédifférenciation, dans M. Grawitz et J. Leca (dir.), Traité de science politique. III. L'action politique, Paris, 1985, p. 643-682.

Birnbaum 2011 = P. Birnbaum, Défense de l'Etat «fort ». Réflexions sur la place du religieux en France et aux Etats-Unis, dans Revue française de sociologie, N52, Vol. 3, 2011, p. 559578.

Birnbaum 2015 = P. Birnbaum, Les désarrois d'un fou de l'Etat. Entretiens avec Jean Baumgarten et Yves Déloye, Paris, 2015.

Burgat 1995 = F. Burgat, L'islamisme en face, Paris, 1995.

Cohen - Lacroix - Riutort 2015 = A. Cohen, B. Lacroix et P. Riutort, Qu'est-ce que la science politique?, dans A. Cohen, B. Lacroix et P. Riutort (dir.), Nouveau manuel de science politique, Paris, 2015, p. 21-27.

Côté $2003=$ P. Côté, Autorité publique, pluralisation et sectorisation religieuse en modernité tardive, dans Archives de sciences sociales des religions, Vol. 121, 2003, p. 19-39.

Crettiez $2016=\mathrm{X}$. Crettiez, Penser la radicalisation. Une sociologie processuelle des variables de l'engagement violent, dans Revue française de science politique, Vol. 66, N5, 2016, p. 709-726. 
Dargent 2010 = C. Dargent, La population musulmane de France : de l'ombre à la lumière?, dans Revue française de sociologie, Vol. 51, N², 2010. p. 219-246.

De Certeau 1981 = M. De Certeau, Une pratique sociale de la différence : croire, dans Faire croire: modalités de la diffusion et de la réception des messages religieux du XIIe au XVe siècle. Table ronde organisée par l'Ecole française de Rome en collaboration avec l'Institut d'histoire médiévale de l'Université de Padoue (Rome, 22-23 juin 1979), Rome, 1981, p. 363383.

De Galembert 2008 = C. De Galembert, Catholicisme et nation au miroir d'une cathédrale, dans A. Capelle-Pogacean, P. Michel et E. Pace (dir.), Religion(s) et identité(s) en Europe. L'épreuve du pluriel, Paris, 2008, p. 255-279.

De Galembert $2014=$ C. De Galembert et M. Koenig, Gouverner le religieux avec des juges. Introduction, Revue française de science politique, Vol. 64, N, 2014, p. 631-645.

Della Sudda 2010 = M. Della Sudda, La politique malgré elles. Mobilisations féminines catholiques en France et en Italie (1900-1914), dans Revue française de science politique, Vol. 60, $\mathrm{N}^{\circ} 1,2010$, p. 37-60.

Déloye $1994=$ Y. Déloye, Ecole et citoyenneté. L'individualisme républicain de Jules Ferry à Vichy: controverses, Paris, 1994.

Donegani 1993 = J.-M. Donegani, La liberté de choisir. Pluralisme religieux et pluralisme politique dans le catholicisme français contemporain, Paris, 1993.

Dosse 1997 = F. Dosse, L'Empire du sens. L'humanisation des sciences humaines, Paris, 1997.

Draï 1985 = R. Draï, Science politique et psychanalyse, dans M. Grawitz et J. Leca (dir.), Traité de science politique. III. L'action politique, Paris, 1985, p. 141-163. 
Dubois 2003 = V. Dubois, La sociologie de l'action publique. De la sociohistoire à l'observation des pratiques (et vice-versa), dans P. Laborier et D. Trom (dir.), Historicités de l'action publique, Paris, 2003, p. 347-364.

Etienne 1976 = B. Etienne, Algérie, cultures et révolution, Paris, 1976.

Foret 2006 = F. Foret, Quels présupposés pour la démocratie européenne ? Regards croisés sur le rôle du religieux, dans Politique européenne, $\mathrm{N}^{\circ} 19$, 2006, p. 117-141.

Foret 2008 = F. Foret, Légitimer l'Europe. Pouvoir et symbolique à l'ère de la gouvernance, Paris, 2008.

Foret - Itçaina 2008 = F. Foret et X. Itçaina, Dieu loin de Bruxelles. L'européanisation informelle du religieux, dans Politique européenne, N²4, 2008, p. 5-20.

Frégosi - Willaime 2001 = F. Frégosi et J.-P. Willaime (dir.), Le Religieux dans la commune. Les régulations locales du pluralisme religieux en France, Genève, 2001.

Gauchet 1985 = M. Gauchet, Le désenchantement du monde, Paris, 1985.

Geisser 2012 = V. Geisser, La «question musulmane » en France au prisme des sciences sociales. Le savant, l'expert et le politique, dans Cahiers d'études africaines, Vol. 2, №206207, 2012, p. 351-366.

Habermas 2006 = J. Habermas, Religion in the Public Sphere, dans European Journal of Philosophy, Vol. 14, 2006, p. 1-25.

Hervieu-Léger 1973 = D. Hervieu-Léger, De la mission à la protestation. L'évolution des étudiants chrétiens en France (1965-1970), Paris, 1973.

Hervieu-Léger 1993 = D. Hervieu-Léger, La religion pour mémoire, Paris, 1993.

Hervieu-Léger 2000 = D. Hervieu-Léger, La sociologie des religions en France. De la sociologie de la sécularisation à la sociologie de la modernité religieuse, dans J.-M. Berthelot (dir.), La sociologie française contemporaine, Paris, 2000, p. 241-250. 
Jouanneau - Raison du Cleuziou 2012 = S. Jouanneau et Y. Raison du Cleuziou, Introduction, dans Genèses, Vol. 3, Nº8, 2012, p. 2-5.

Jouanneau $2013=\mathrm{S}$. Jouanneau, Les imams en France. Une autorité religieuse sous contrôle, Marseille, 2013.

Kantorowicz 1989 = E. Kantorowicz, Les deux corps du roi. Essai sur la théologie politique au Moyen Âge, Paris, 1989 (1 $1^{\text {ère } e ́ d . ~ 1957) . ~}$

Kepel 1984 = G. Kepel, Le Prophète et Pharaon. Les mouvements islamistes dans l'Egypte contemporaine, Paris, 1984.

Kepel 1987 = G. Kepel, Les banlieues de l'islam. Naissance d'une religion en France, Paris, 1987.

Kettell 2008 = S. Kettell, Has Political Science Ignored Religion?, dans PS : Political Science and Politics, Vol. 45, $\mathrm{N}^{\circ} 1,2012$, p. 93-100.

Lagroye 1985 = J. Lagroye, La légitimation, dans M. Grawitz et J. Leca (dir.), Traité de science politique. I. La science politique, science sociale. L'ordre politique, Paris, 1985, p. $395-467$.

Lagroye $2006=\mathrm{J}$. Lagroye, La vérité dans l'Eglise catholique. Contestations et restauration d'un régime d'autorité, Paris, 2006.

Lagroye 2009 = J. Lagroye, Appartenir à une institution. Catholiques en France aujourd'hui, Paris, 2009.

Lagroye - Offerlé 2010 = J. Lagroye et M. Offerlé (dir.), Sociologie de l'institution, Paris, 2010.

Legendre 1976 = P. Legendre, Jouir du pouvoir. Traité de la bureaucratie patriote, Paris, 1976.

Leveau - Kepel 1988 = R. Leveau et G. Kepel (dir.), Les musulmans dans la société française, Paris, 1988. 
Lorcerie 1996 = F. Lorcerie, Lä̈cité 1996. La République à l'école de l'immigration ?, dans Revue française de pédagogie, $\mathrm{N}^{\circ} 117,1996$, p. 53-86.

Michel 1993 = P. Michel, Pour une sociologie des itinéraires de sens : une lecture politique du rapport entre croire et institution, dans Archives des sciences sociales des religions, N. 82, 1993, p. 223-238.

Michel 1997 = P. Michel (dir.), Religion et démocratie, Paris, 1997.

Michelat - Simon 1977 = G. Michelat et M. Simon, Classe, religion et comportements politiques, Paris, 1977.

Monnerot - Arendt $2012=\mathrm{J}$. Monnerot et H. Arendt, Le communisme peut-il être pensé dans le registre de la religion, dans A. Caillé (dir.), Qu'est-ce que le religieux? Religion et politique, Paris, 2012, p. 40-46.

Ollion 2010 = E. Ollion, La secte sécularisée : étude d'un processus de requalification conceptuelle, dans Genèses, Vol. 78, 2010, p. 25-47.

O’Miel - Talpin $2015=$ J. O’Miel et J. Talpin, Espace et conflits dans la participation. Luttes symboliques et matérialité d'une controverse autour de la localisation d'une mosquée à Florence, dans Lien social et politiques, $\mathrm{N}^{\circ} 73,2015$, p. 33-52.

Ozzano - Giorgi 2015 = L. Ozzano et A. Giorgi, European Culture Wars and the Italian Case. Which Side Are You On ?, New York, 2015.

Palard 1996 = J. Palard (dir.), Le gouvernement de l'Eglise catholique. Synodes et exercice du pouvoir, Paris, 1996.

Pina $2000=\mathrm{C}$. Pina, Les évêques français face au monde moderne : l'exemple du rapport Dagens, dans P. Bréchon, B. Duriez et J. Ion (dir.), Religion et action dans l'espace public, Paris, 2000, p. 91-105.

Portier 2006 = P. Portier, La pensée de Jean-Paul II. La critique du monde moderne, Paris, 2006. 
Portier $2010=\mathrm{P}$. Portier, Modernités plurielles ? Une analyse longitudinale des régimes de régulation du croire dans les démocraties stabilisées, in M. Milot, P. Portier et J.-P. Willaime (dir.), Pluralisme religieux et citoyenneté, Rennes, 2010, p. 220-255.

2016 = P. Portier, L'Etat et les religions en France. Une sociologie historique de la laïcité, Rennes, 2016.

Raison du Cleuziou 2010 = Y. Raison du Cleuziou, Des fidélités paradoxales. Recomposition des appartenances et militantisme institutionnel dans une institution en crise, dans J. Lagroye et M. Offerlé (dir.), Sociologie de l’institution, Paris, 2010, p. 267-290.

Raison du Cleuziou 2015 = Y. Raison du Cleuziou, Le pouvoir religieux et l'Etat en France, dans A. Cohen, B. Lacroix et P. Riutort (dir.), Nouveau manuel de science politique, Paris, 2015, p. 292-298.

Rousset $2016=$ M. Rousset, Quand la sociologie entre en religion, in Le Monde, 17 mars 2016.

Roy 1985 = O. Roy, L'Afghanistan. Islam et modernité politique, Paris, 1985.

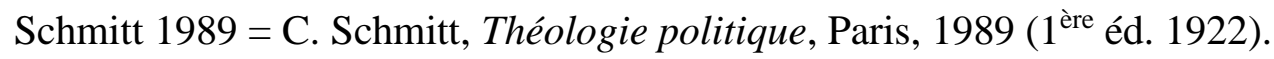

Taylor 2011 = C. Taylor, L'âge séculier, Paris, 2011.

Teinturier $2013=\mathrm{S}$. Teinturier, Coéducation, gémination, mixité. Un débat catholique dans l'entre-deux-guerres, dans F. Rochefort et M.E. Sanna (dir.), Normes religieuses et genre. Mutations, résistances et reconfiguration. XIXe-XXIe siècle, Paris, 2013, p. 87-96.

Tozy 2009 = M. Tozy, Mutations du champ politico-religieux marocain, dans F. Frégosi (dir.), Bruno Etienne, le fait religieux comme fait politique, La Tour d'Aigues, 2009, p. 113134.

Tozy 2013 = M. Tozy, Des oulémas frondeurs à la bureaucratie du croire. Les péripéties d'une restructuration annoncée du champ religieux au Maroc, dans B. Hibou (dir.), La bureaucratisation néolibérale, Paris, 2013, p. 129-154. 
Turina 2013 = I. Turina, Chiesa e biopolitica. Il discorso cattolico su famiglia, sessualità e vita umana da Pio IX a Benedetto XVI, Milan, 2013.

Voegelin 1994 = E. H. W. Voegelin, Les religions politiques, Paris, 1994.

Willaime 2004 = J.-P. Willaime, Europe et religions. Les enjeux du XXIe siècle, Paris, 2004.

Zambiras $2014=$ A. Zambiras, La politique inspirée. Controverses publiques et religion aux Etats-Unis, Paris, 2014.

\footnotetext{
${ }^{1}$ Nous remercions Marc-Olivier Del Grosso, Audrey Freyermuth, Diletta Guidi et Françoise Lorcerie pour leurs relectures et suggestions, tout en revendiquant notre seule responsabilité pour les défauts et limites de ce texte.

${ }^{2}$ Amiraux 2012a, p. 336-337.

${ }^{3}$ Baubérot - Milot 2011, p. 177-194.

${ }^{4}$ Geisser 2012.

${ }^{5}$ Le politiste Xavier Crettiez affirme, d'après les résultats d'une enquête de Benjamin Ducol, que 3\% des publications sur la radicalisation dans les principales revues anglo-saxonnes de science politique sur les conflits datent des années 1980-1999, et 77\% sont postérieures à 2006. Voir Crettiez 2016, p. 709.

${ }^{6}$ Turina 2013.

${ }^{7}$ Altglas 2012, p. 38-40.

${ }^{8}$ Cité dans Rousset 2016.

${ }^{9}$ Kettell 2012.

${ }^{10}$ Kantorowicz 1989.

${ }^{11}$ Schmitt 1989.

${ }^{12}$ Voegelin 1994.

${ }^{13}$ Bellah 1973.

${ }^{14}$ Monnerot - Arendt 2012.

${ }^{15}$ Aron 1965.

${ }^{16}$ Cohen - Lacroix - Riutort 2015, p. 24-25.

${ }^{17}$ Legendre 1976, p. 15.

${ }^{18}$ Michelat - Simon 1977.

${ }^{19}$ Dargent 2010.

${ }^{20}$ Hervieu-Léger 1973.
} 
${ }^{21}$ Hervieu-Léger 1993, p. 35-64.

${ }^{22}$ Raison du Cleuziou 2015, p. 295-297.

${ }^{23}$ Dosse 1997, p. 335-367.

${ }^{24}$ Gauchet 1985 .

${ }^{25}$ Voir Lagroye 1985 ; Draï 1985 ; Birnbaum 1985.

${ }^{26}$ Burgat 1995.

${ }^{27}$ Roy 1985.

${ }^{28}$ Etienne 1976.

${ }^{29}$ Le terme « islamisme » dans cette acception apparait dans les travaux de Bruno Etienne et Mohamed Tozy sur le culte des saints à Casablanca en 1978-1979. Voir Tozy 2009.

${ }^{30}$ Kepel 1984.

${ }^{31}$ Kepel 1987 ; Leveau - Kepel 1988.

${ }^{32}$ Geisser 2012, p. 353-359.

${ }^{33}$ Michel 1993.

${ }^{34}$ Michel 1997.

${ }^{35}$ Voir par exemple Baubérot 2014, p. 169-170.

${ }^{36}$ Dans ses travaux sur le catholicisme, voir notamment Palard 1996.

${ }^{37}$ Françoise Lorcerie mène alors, au croisement de la sociologie politique et de la sociolinguistique, des travaux sur l'islam dans les programmes, l'ethnicité et la laïcité à l'école. Voir Lorcerie 1996.

${ }^{38}$ Voir Birnbaum 2015, p. 133-160.

${ }^{39}$ Déloye 1994.

${ }^{40}$ Donegani 1993, p. 461.

${ }^{41}$ Voir notamment C. Pina 2000.

${ }^{42}$ Altglas 2010.

${ }^{43}$ Geisser 2012, p. 359-362 ; Amiraux 2012b, p. 145-146.

${ }^{44}$ Habermas 2006 ; Taylor 2011.

${ }^{45}$ Voir Lagroye - Offerlé 2010.

${ }^{46}$ Willaime 2004.

${ }^{47}$ Altglas 2010, p. 493-494.

${ }^{48}$ Voir notamment Portier 2006. 
${ }^{49}$ Portier 2010; Portier 2016.

${ }^{50}$ Foret 2006, p. $138-139$.

${ }^{51}$ Foret 2008.

${ }^{52}$ Foret - Itçaina 2008.

${ }^{53}$ Amiraux 2012b, p. 148-149.

${ }^{54}$ Voir AFSP 2007.

${ }^{55}$ Lagroye 2006, p. 225-257.

${ }^{56}$ Lagroye 2009, p. 49.

${ }^{57}$ Della Sudda 2010.

${ }^{58}$ Jouanneau - Raison du Cleuziou 2012.

${ }^{59}$ Raison du Cleuziou 2010 ; Teinturier 2013.

${ }^{60}$ Zambiras 2014.

${ }^{61}$ Hervieu-Léger 2000, p. 249.

${ }^{62}$ Voir notamment Birnbaum 2011.

${ }^{63}$ Côté 2003.

${ }^{64}$ Frégosi - Willaime 2001.

${ }^{65}$ De Galembert 2008.

${ }^{66}$ Tozy 2013.

${ }^{67}$ Béraud - De Galembert - Rostaing 2016.

${ }^{68}$ Dubois, 2003.

${ }^{69}$ Ollion 2010.

${ }^{70}$ Jouanneau 2013, p. 257-258.

${ }^{71}$ Voir AFSP 2013.

${ }^{72}$ O’Miel - Talpin 2013.

${ }^{73}$ De Galembert - Koenig 2014.

${ }^{74}$ Altglas 2012.

${ }^{75}$ De même, la religion dans les relations internationales est rarement abordée en-dehors de considérations sur la doctrine et la géopolitique, à l'exception de travaux comme ceux de Marie Balas sur la communauté de Sant'Egidio et la diplomatie religieuse. Voir Balas 2013.

${ }^{76}$ Voir par exemple pour l'Italie, Ozzano - Giorgi 2015. 
${ }^{77}$ Kettell 2008, p. 95-96.

${ }^{78}$ Voir l'enquête de Julien Beaugé et Abdellali Hajjat sur les membres du Haut Conseil à l'Intégration et la production d'expertise sur l'islam et la laïcité en France ; Beaugé - Hajjat 2014.

${ }^{79}$ De Certeau 1981, p. 381. 